\title{
VI. Italienischer Krebskongreß
}

Vom 10.-12. Marz 1950 hielt die italienische Liga fur Krebsbekampfung unter dem Vorsitz von Prof. P. Rondoni ihren 6. KongreB in Cremona ab. Nach einfuhrenden Wor-ten von Pentimalli (Rom) iiber das Wesen des Krebses, sprach Natale (Mailand) uber die verschiedenen Methoden zur Friihdiagnose des Uteruskrebses. In seinem Vortrag iiber die Pathologic des Magenkrebses weist Bucalossi (Mailand) u. a. auf die diagnostischen Schwierigkeiten hin, durch welche die Patienten sehr oft zu spat der geeigneten Behandlung zugefiihrt werdon, und auf die pradisponierenden Faktoren, wie die verschiedenen For-men der Gastritis, die in etwa 14\% als Ursachen des Krebses anzusehen sind. Sirtori (Mailand) gibt, z. T. auf Grund eigener Untersuchungen, einen Uberblick iiber die Be-ziehungen zwischen Krebs und Hormonen, besonders den Sexualhormonen. Der Phjrsiker Caldirola (Mailand) befafit sich mit der Struktur des Atomkernes und den radioaktiven Prozessen. Radioaktive Isotope gelangen in der Onkologie zur Anwendung: 1. zum Studium des Entstehungsmechanismus von Knoohentumoren durch Beryllium, 2. in der Diagnostik zur Lokalisierung von Hirntumoren und 3. therapeutisch. Die Frage: Be-stehen irgendwelche Gefahren in der Strahlentherapie der Krebse ? wird von Ratti (Pavia) auf Grund der heutigen Erfahrungen verneinend beantwortet. Bei riehtiger Anwendung der Strahlentechnik sind keine Schadigungen zu erwarten, so wie man sie vom Tier-experiment und vom Berufskrebs her kennt. Unvermeidlich sind hingegen die mitunter als Strahlenfolge auftretenden trophischon VToranderungen der Haut und Schleimhaut, die jedoch den kurativen Wert der Strahlentherapie in keiner Weise beeintrachtigen. Anschliefiend beriehtete Lelli (Rom) kurz iiber seine bisherigen Resultate der Darstel-lung des Kollagens verschiedener Formen von Mammatumoren im Elektronenmikroskop. Uber den Gebrauch der Strahlenbehandlung und antimitotischer und cytostatischer Substanzen in der Behandlung der Blutkrankheiten referiert Fieschi (Siena). Auf die Beziehungen zwischen Leukamien und bosartigen Tumoren wird nicht eingegangen, es wird nur daran erinnert, daB viele antileukamische Heilmittel ebenso aktiv wie gewisse «Antineoplastica» sind und ebenso wie diese experimentell Krebs erzeugen konnen. Es wird die therapeutische Anwendung des Urethans, der Nitrogen Mustards und der Anti-folica besprochen, deren Verwendung wegen ihrer hohen Toxizitat nur dann erlaubt ist, 192

\section{Libri}

vvenn sich die Rontgentherapie als ungeniigend erweist. In seinem Referat iiber den Rektumkrebs setzt sich Margottini (Rom) hauptsachlich mit der operativen Technik auseinander. Die Diagnose und Therapie der Lungentumoren werden auf Grund der eigenen Erfahrungen an 145 Fallen von Valdoni (Rom) beleuchtet, wahrend Zelioli (Cremona) iiber den Krebs als soziale Krankheit und die Organisierung der Krebsbekampfung in Italien und anderen Landern referiert. Pietrantoni (Mailand) beriehtet iiber die Atio-logie, Symptomatologie und Therapie der Pharynxund Larynxkreb.se, wobei er sich sehr fiir eine kombinierte chirurgisch-radiotherapeutische Behandlung, besonders der Tumoren der Zungenbasis, einsetzt. Abschliefiend gibt Fasiani (Mailand) einen Uber-blick iiber den augenblicklichen Zustand der Chirurgie der Hirntumoren. U. Cocchi, Zurich 


\section{Libri}

Bucher, O.: Histologie unci mikroskopische Anatomie des Menschen mit Beriicksichtigung der Histophi/siologie unci der mikroskopischen Diagnostik. Medizinischer Verlag Hans Huber, Bern. 468 Seiten, 20 Tabellen, 352 teils mehrfarbige Abbildungen. Preis ge-bunden Fr. 65.70, broschiert Fr. 59.50. 1948.

Vor dem zweiten Weltkrieg waren deutsche Lehr- und Handbiicher in der Schweiz weit verbreitet, denn es handelte sich in kultureller und wissenschaftlicher Beziehung urn eine Einheit, der auch wir Deutschschweizer angehorten. Das hat sich geandert, und der groBe Mangel an deutschsprachigen medizinischen Lehrbiichern zwang uns, auch in der Schweiz eigene Bucher fiir die Studenten zu verfassen und durch Schweizer Verlage herauszubringen. So ist das vorliegende Lehrbuch der Histologie und mikroskopischen Anatomie im medizinischen Verlag Hans Huber, Bern 1948 erschienen. Es ist sowohl nach Inhalt als auch nach Ausstattung ein Werk, das jedem Vergleich standhalt. Die neuesten Ergebnisse der wissenschaftlichen Forschung sind beriicksichtigt und viele Literaturhinweise gegeben. Die Vorlagen zu den rund 350, zum Teil farbigen Abbildungen stammen zum GroBteil von eigenen Priiparaten oder sind instruktive Schemata, die vom Verfasser selber entworfen worden sind. Zytomorphologie und Zytophysiologie werden auf etwa 50 Seiten dargestellt, wobei auch der sublichtmikroskopische Bau erortert wird. Zu kurz und den heutigen Kenntnissen nicht ganz entsprechend ist die Schilderung der Meiose. Es werden nicht Merkmale, sondern Gene ausgetauscht. Der angehende Medizinstudent wird sich iiber diese Dinge freilich nicht in einem Histologiebuch orientieren, sondern in einem Lehrbuch der Zytologie auf genetischer Grundlage. Rund 100 Seiten sind der Histologie gewidmet, wahrend die mikroskopische Anatomie rund 300 Seiten umfaBt und nach Organsystemen gegliedert ist. Wertvoll fiir den Studenten sind die Kapitel iiber die Differentialdiagnose der verschiedenen Gewebe und ihre Bedeutung fiir die mikroskopische Organdiagnostik. Didaktisch geschickt ist jeweils auch die Zusammenfassung der Baueigentiimlichkeiten jedes einzelnen Organsystems in differentialdiagnostischer Hinsicht. In einem Anhang werden die drei gebrauchlichsten histologischen Farbungen angefiihrt und das Vorgehen iiber das Herstellen eines histologischen Praparates geschildert. 20 instruktive Tabellen erganzen den Text. Der Preis ist niedrig. Das Lehr buch fiillt eine zur Zeit bestehende Liicke vollstandig aus und kann auch den Krebsforschern und Krebstherapeuten zum Studium empfohlen werden, denn die Morphologie der Krebse baut sich nach wie vor auf der Xormalhistologie auf und ist ein unentbehrliches Hilfsmittel fiir unsere Tatigkeit. H. R. Schinz, Zurich. 